\title{
DRAFT
}

\section{Partitioning Complete Multipartite Graphs by Monochromatic Trees}

\author{
Atsushi Kaneko, M.Kano ${ }^{1}$ and Kazuhiro Suzuki ${ }^{1}$ \\ ${ }^{1}$ Department of Computer and Information Sciences \\ Ibaraki University, Hitachi 316-8511 Japan \\ e-mail: kano@cis.ibaraki.ac.jp and tutetuti@dream.com
}

April 27, 2004

\begin{abstract}
The tree partition number of an $r$-edge-colored graph $G$, denoted by $t_{r}(G)$, is the minimum number $k$ such that whenever the edges of $G$ are colored with $r$ colors, the vertices of $G$ can be covered by at most $k$ vertex-disjoint monochromatic trees. We determine $t_{2}\left(K\left(n_{1}, n_{2}, \ldots, n_{k}\right)\right)$ of the complete $k$-partite graph $K\left(n_{1}, n_{2}, \ldots, n_{k}\right)$. In particular, we prove that $t_{2}(K(n, m))=\left\lfloor(m-2) / 2^{n}\right\rfloor+2$, where $1 \leq n \leq m$.
\end{abstract}

\section{Introduction}

We consider finite graphs without loops or multiple edges. The complete $k$-partite graph $K\left(n_{1}, n_{2}, \ldots, n_{k}\right)$ has the vertex set $V_{1} \cup V_{2} \cup \cdots \cup V_{k}$ such that $V_{i} \cap V_{j}=\emptyset$ and $\left|V_{i}\right|=n_{i}$ for every $1 \leq i<j \leq k$, and the edge set $\left\{x_{i} x_{j} \mid x_{i} \in V_{i}, x_{j} \in V_{j}, 1 \leq i<j \leq k\right\}$. We often denote by $K(n, m)$ the complete bipartite graph with partite sets $X$ and $Y$, where $|X|=n$ and $|Y|=m$.

The tree partition number of an $r$-edge-colorings of a graph $G$, denoted by $t_{r}(G)$, which was introduced by Erdös, Gyárfás and Pyber [1], is the minimum $k$ such that whenever the edges of $G$ are colored with at most $r$ colors, the vertices of $G$ can be covered by at most $k$ vertex-disjoint monochromatic trees. Moreover it was conjectured in [1] that the tree partition number of an $r$-edge-colored complete graph is $r-1$.

It is well-known that either a graph or its complement is connected. This is equivalent to the fact that every 2-edge-colored complete graph has a monochromatic spanning tree, which implies that $t_{2}\left(K_{n}\right)=1$, and thus the conjecture is true for $r=2$. The conjecture was proved for $r=3$ in [1]. Recently Haxell and Kohayakawa [2] obtained the following Theorem A, which implies that $t_{r}\left(K_{n}\right) \leq r$ if $n$ is sufficiently large. 
Theorem A ([2]). $\quad$ The vertex set of the complete graph $K_{n}$ whose edges are colored with $r$ colors can be covered by at most $r$ vertex-disjoint monochromatic trees with different colors provided $n \geq 3 r^{4} r !(1-1 / r)^{3(1-r)} \log r$.

It is mentioned in [3] that the following theorem can be proved by similar arguments given in $[2]$.

Theorem B ([3]). If $n$ is sufficiently large, $t_{r}(K(n, n)) \leq 2 r$.

For related results of Ramsey partitioning type, see [4],[5].

When we consider 2-edge-colored graphs, we always assume that all their edges are colored red or blue. So a monochromatic tree of 2-edge-colored graph can be called a red tree or a blue tree.

In this paper we first consider $t_{2}\left(K\left(n_{1}, N_{2}, \ldots, n_{k}\right)\right)$, and prove the following theorem in section 2 .

Theorem 1. Let $n_{1}, n_{2}, \ldots, n_{k}(2 \leq k)$ be integers such that $1 \leq n_{1} \leq n_{2} \leq \cdots \leq n_{k}$, and let $n=n_{1}+n_{2}+\cdots+n_{k-1}$ and $m=n_{k}$. Then

$$
t_{2}\left(K\left(n_{1}, n_{2}, \ldots, n_{k}\right)\right)=\left\lfloor\frac{m-2}{2^{n}}\right\rfloor+2 .
$$

Note that $K(1,1, \ldots, 1)$ (i.e. $\left.n_{k}=1\right)$ is isomorphic to a complete graph, and so it has a monochromatic spanning tree. On the other hand, the right side of (1) is equal to one as $\left\lfloor(m-2) / 2^{n}\right\rfloor=-1$.

Next we consider a related problem. Every complete multipartite graph that is not a complete graph has two non-adjacent vertices $x$ and $y$. If all the edges incident with $x$ are colored red and all the edges incident with $y$ are colored blue, then such a 2-edge-colored complete multipartite graph has no monochromatic spanning trees. Therefore, in order to guarantee the existence of monochromatic spanning tree in a 2-edge-colored complete multipartite graph $G$, it is necessary to assume that for every vertex $v$ of $G$, at least one red edge and at least one blue edge are incident with $v$. However this is not sufficient. Consider a complete bipartite graph $K(n, m)(2 \leq n \leq m)$ with partite sets $X$ and $Y$, and choose two vertices $x \in X$ and $y \in Y$. If the edge $x y$ and all the edges joining $X-x$ to $Y-y$ are colored red and all the other edges are colored blue, then $K(n, m)$ has no monochromatic spanning trees.

Our second theorem shows that the bipartite case is exceptional.

Theorem 2. Let $k \geq 3$ be an integer. If the edges of the complete $k$-partite graph $K\left(n_{1}, n_{2}, \ldots, n_{k}\right)$ are colored red or blue in such a way that for every vertex $v$, at least one red edge and at least one blue edge are incident with $v$, then $K\left(n_{1}, n_{2}, \ldots, n_{k}\right)$ has a monochromatic spanning tree.

We prove Theorem 2 in section 3. 


\section{Proof of Theorem 1}

For a graph $G$, we denote by $V(G)$ and $E(G)$ the set of vertices and the set of edges of $G$, respectively. An edge joining a vertex $x$ to a vertex $y$ is denoted by $x y$ or $y x$. For two disjoint sets $X$ and $Y$ of vertices of a graph $G$, we denote by $E(X, Y)$ the set of edges of $G$ joining a vertex in $X$ to a vertex in $Y$, in fact, in all our proofs we use $E(X, Y)$ to denote something stronger: the complete bipartite graph with vertex classes $X$ and $Y$, which is in every case a subgraph of the graph we are considering. For a set $X$ of vertices of $G$, we denote by $\langle X\rangle$ the subgraph of $G$ induced by $X$. For a subgraph $H$ and a set $X$ of vertices of $G$, we say that $H$ covers $X$ or $X$ is covered by $H$ if $X \subseteq V(H)$.

In order to prove Theorem 1, we need three lemmas.

Lemma 1. Let $k \geq 3$ be an integer, and $\left\{n_{1}, n_{2}, \ldots, n_{k}\right\}$ be a set of positive integers such that $n_{k} \geq n_{i}$ for all $1 \leq i \leq k-1$ and $n_{1}+n_{2}+\cdots+n_{k-1}>n_{k} \geq 2$. Then the set of indices $\{1,2, \ldots, k\}$ can be partitioned into two disjoint subsets $I \cup J$ so that

$$
n \leq m \leq 2^{n}+1, \quad \text { where } n=\sum_{i \in I} n_{i} \quad \text { and } m=\sum_{j \in J} n_{j} .
$$

In particular, the complete $k$-partite graph $K\left(n_{1}, n_{2}, \ldots, n_{k}\right)$ contains a spanning complete bipartite graph $K(n, m)$ such that $n \leq m \leq 2^{n}+1$.

Proof. Without loss of generality, we may assume that $n_{1} \leq n_{2} \leq \cdots \leq n_{k}$. Take an integer $t(1 \leq t \leq k-2)$ such that

$$
\begin{aligned}
n_{t}+n_{t+1}+n_{t+2}+\cdots+n_{k-1} & >n_{k}+n_{1}+n_{2}+\cdots+n_{t-1} \quad \text { and } \\
n_{t+1}+n_{t+2}+\cdots+n_{k-1} & \leq n_{k}+n_{1}+n_{2}+\cdots+n_{t-1}+n_{t} .
\end{aligned}
$$

Let $a=n_{t}, \quad b=n_{t+1}+\cdots+n_{k-1}$ and $c=n_{k}+n_{1}+n_{2}+\cdots+n_{t-1}$. Then

$$
a \leq b, \quad 2 \leq n_{k} \leq c<a+b \quad \text { and } \quad b \leq a+c .
$$

It suffices to show that $b \leq a+c \leq 2^{b}+1$ or $c<a+b \leq 2^{c}+1$ by letting either $n=b$ and $m=a+c$ or $n=c$ and $m=a+b$.

If $b \leq 2$, then $a+b \leq b+b \leq 4 \leq 2^{c}+1$. Therefore we may assume that $b \geq 3$. It is easy to prove that $2^{x}+1 \geq 3 x$ for $x \geq 3$. Hence $2^{b}+1 \geq 3 b \geq a+a+b>a+c$. Consequently the lemma is proved.

The following Lemmas 2 and 3, which determine $t_{2}(K(n, m))$, are essential parts of the proof of Theorem 1 .

Lemma 2. Let $n$ and $m$ be integers such that $1 \leq n \leq m$. Then the vertices of 2-edgecolored complete bipartite graph $K(n, m)$ can be covered by at most

$$
\left\lfloor\frac{m-2}{2^{n}}\right\rfloor+2
$$

vertex-disjoint monochromatic trees. 
Proof. Let $X=\left\{x_{1}, x_{2}, \ldots, x_{n}\right\}$ and $Y=\left\{y_{1}, y_{2}, \ldots, y_{m}\right\}$ be the partite sets of $K(n, m)$. Suppose that all the edges of $K(n, m)$ are colored red or blue. For an $n$-tuple $\left(a_{1}, \ldots, a_{n}\right)$ such that $a_{i} \in\{r, b\}$ for every $i$, we define

$$
Y\left(a_{1}, a_{2}, \ldots, a_{n}\right)=\left\{y \in Y \mid y x_{i} \text { is red or blue according as } a_{i}=r \text { or } a_{i}=b\right\} .
$$

In particular, $Y(r, r, \ldots, r)$ and $Y(b, b, \ldots, b)$ denote the sets of vertices $y \in Y$ such that all the edges incident with $y$ are red or blue, respectively. We abbreviate $Y(r, r, \ldots, r)$ to $Y(r)$ and $Y(b, b, \ldots, b)$ to $Y(b)$. Similarly, we denote by $X(r)$ and $X(b)$ the sets of vertices in $x \in X$ such that all the edges incident with $x$ are red or blue, respectively.

We begin with an observation on $\left(a_{1}, a_{2}, \ldots, a_{n}\right)$, which will be often used. If there exists an $n$-tuple $\left(a_{1}, a_{2}, \ldots, a_{n}\right)$ such that $Y\left(a_{1}, a_{2}, \ldots, a_{n}\right)=\emptyset, a_{s}=r$ and $a_{t}=b$ for some $s \neq t$, then by letting $X_{r}=\left\{x_{i} \in X \mid a_{i}=r\right\} \ni x_{s}$ and $X_{b}=\left\{x_{i} \in X \mid a_{i}=b\right\} \ni x_{t}$, $Y$ can be partitioned into

$$
Y=Y_{r} \cup Y_{b}, \quad Y_{r} \cap Y_{b}=\emptyset,
$$

where the two subsets $Y_{r}$ and $Y_{b}$ are defined as

$$
\begin{aligned}
& Y_{r}=\left\{y \in Y \mid y x_{i} \text { is red for some } x_{i} \in X_{b}\right\}, \\
& Y_{b}=\left\{y \in Y \mid y x_{i} \text { is blue for all } x_{i} \in X_{b} \text {, and } y x_{j} \text { is blue for some } x_{j} \in X_{r}\right\} .
\end{aligned}
$$

It is clear that $Y_{r} \cap Y_{b}=\emptyset$ and $Y-\left(Y_{r} \cup Y_{b}\right)=Y\left(a_{1}, a_{2}, \ldots, a_{r}\right)=\emptyset$, and thus the partition given in (3) is well-defined. (see Figure 1)

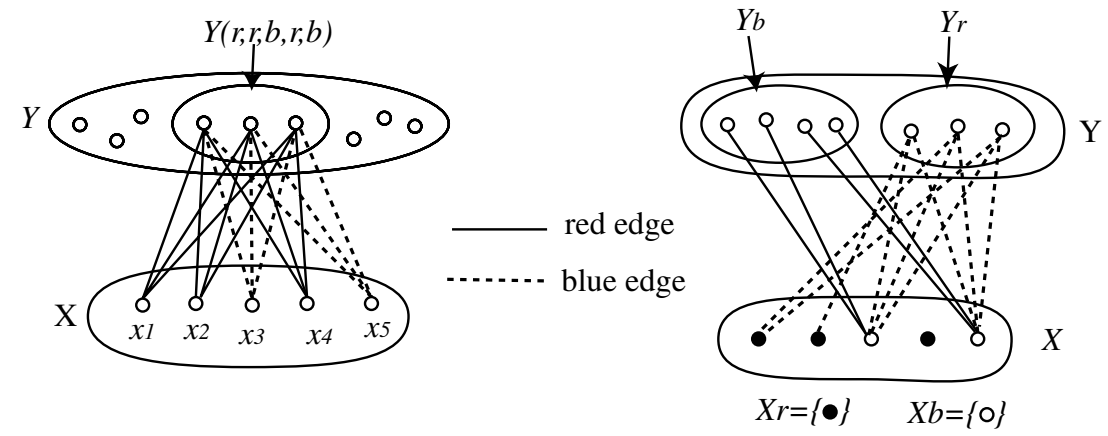

Figure 1: $Y\left(a_{1}, \ldots, a_{5}\right), Y_{r}$ and $Y_{b}$ for $\left(a_{1}, \ldots, a_{5}\right)=(r, r, b, r, b)$

We consider four cases.

Case 1. $Y(r) \neq \emptyset, Y(b) \neq \emptyset$, and for some other $n$-tuple $\left(a_{1}, a_{2}, \ldots, a_{n}\right)$, $Y\left(a_{1}, a_{2}, \ldots, a_{n}\right)=\emptyset$.

For the $n$-tuple $\left(a_{1}, a_{2}, \ldots, a_{n}\right)$ with $Y\left(a_{1}, a_{2}, \ldots, a_{n}\right)=\emptyset$, we get the partition (3). Then $Y(r) \subseteq Y_{r}$ and $Y(b) \subseteq Y_{b}$, and the induced subgraph $\left\langle Y_{r} \cup X_{b}\right\rangle$ contains a spanning red tree $T r$, and the induced subgraph $\left\langle Y_{b} \cup X_{r}\right\rangle$ contains a spanning blue tree $T_{b}$. Hence the set of vertices of $K(n, m)$ is covered by two monochromatic trees $T_{r}$ and $T_{b}$. 
Case 2. Either $Y(r)=\emptyset$ and $Y(b) \neq \emptyset$, or $Y(r) \neq \emptyset$ and $Y(b)=\emptyset$.

Without loss of generality, we may assume that $Y(r)=\emptyset$ and $Y(b) \neq \emptyset$. Since $Y(r)=\emptyset$, it follows that for every vertex $y \in Y$, at least one blue edge is incident with $y$. By $Y(b) \neq \emptyset$, it is immediate that $K(n, m)$ has a blue spanning tree.

Case 3. $Y(r)=Y(b)=\emptyset$.

In this case, for every vertex $y \in Y$, at least one red edge and at least one blue edge are incident with $y$. We first assume $X(r)=X(b)=\emptyset$, that is, we assume that for every vertex $x \in X$, at least one red edge and at least one blue edge are incident with $x$. Let $H$ be the subgraph of $K(n, m)$ induced by the red edges of $K(n, m)$. Since $V(H)=V(K(n, m))$, we may assume that $H$ is not connected. Let $C$ be a component of $H$. Then $C$ covers neither $X$ nor $Y$ since otherwise $H$ must contain a spanning tree of $K(n, m)$, which is a contradiction.

Put

$$
A=X \cap V(C), P=X-A, B=Y \cap V(C), \quad Q=Y-B .
$$

Then each of the above four vertex sets is a non-empty set, and all the edges in $E(A, Q) \cup$ $E(P, B)$ are blue, and thus the vertices of $K(n, m)$ can be covered by two blue trees.

If either $X(r)=\emptyset$ and $X(b) \neq \emptyset$, or $X(r) \neq \emptyset$ and $X(b)=\emptyset$, then we can find a blue spanning tree or a red spanning tree of $K(n, m)$, respectively.

We finally assume $X(r) \neq \emptyset$ and $X(b) \neq \emptyset$. For an $m$-tuple $\left(a_{1}, a_{2}, \ldots, a_{m}\right)\left(a_{i} \in\right.$ $\{r, b\})$, we define

$$
X\left(a_{1}, a_{2}, \ldots, a_{m}\right)=\left\{x \in X \mid x y_{i} \text { is red or blue according as } a_{i}=r \text { or } a_{i}=b\right\} .
$$

Then since $n \leq m<2^{m}$, for some $m$-tuple $\left(a_{1}, \ldots, a_{m}\right), X\left(a_{1}, \ldots, a_{m}\right)=\emptyset$. Hence by the same argument as in the proof of Case 1, we can show that the vertices of $K(n, m)$ can be covered by a red tree and a blue tree which are vertex-disjoint.

Case 4. For every $n$-tuple $\left(a_{1}, \ldots, a_{n}\right), Y\left(a_{1}, \ldots, a_{n}\right) \neq \emptyset$.

Let $Z$ be the set of all $Y\left(a_{1}, \ldots, a_{n}\right), t^{\prime}=\min \{|V| \mid V \in Z \backslash\{Y(r), Y(b)\}\}$, and $t=\min \left\{|Y(r)|-1,|Y(b)|-1, t^{\prime}\right\}$. Since $|Z|=2^{n}$ and the union of all the sets $Y\left(a_{1}, \ldots, a_{n}\right)$ is equal to $Y$, we have

$$
t \leq\left\lfloor\frac{m-2}{2^{n}}\right\rfloor .
$$

If $t=|Y(r)|-1$, then $K(n, m)-Y(r)$ has a blue spanning tree since $Y(b) \neq \emptyset$ and for every vertex $y \in Y-Y(r)$, there is at least one blue edge incident with $y$. Therefore $V(K(n, m))$ can be covered by $t+1$ isolated vertices in $Y(r)$ and one blue tree, which implies the lemma holds. Thus we may assume that $t \neq|Y(r)|-1,|Y(b)|-1$.

Suppose that $t=\left|Y\left(a_{1}, \ldots, a_{n}\right)\right|$, where $\left(a_{1}, \ldots, a_{n}\right) \neq(r, r, \ldots, r),(b, b, \ldots, b)$. Then $K(n, m)-Y\left(a_{1}, \ldots, a_{n}\right)$ satisfies the condition of Case 1, and hence the vertices of 
$K(n, m)-Y\left(a_{1}, \ldots, a_{n}\right)$ are covered by two vertex-disjoint monochromatic trees. Therefore $V(K(n, m))$ can be covered by $t$ vertex-disjoint isolated vertices in $Y\left(a_{1}, \ldots, a_{n}\right)$ and two monochromatic trees of $K(n, m)-Y\left(a_{1}, \ldots, a_{n}\right)$, which implies the lemma holds. Consequently the lemma is proved.

Lemma 3. We can color all the edges of the complete bipartite graph $K(n, m)(1 \leq n \leq$ $m)$ red or blue so that the vertices of the resulting 2-edge-colored $K(n, m)$ cannot be covered by fewer than $\left\lfloor(m-2) / 2^{n}\right\rfloor+2$ vertex-disjoint monochromatic trees.

Proof. If $n=1$, then $K(1, m)$ is a star, and $\left\lfloor(m-2) / 2^{n}\right\rfloor+2=\lfloor m / 2\rfloor+1$. We can color $\lfloor m / 2\rfloor$ edges red and $\lceil m / 2\rceil$ edges blue, which implies the lemma holds. Thus we may assume $2 \leq n$.

Let $X=\left\{x_{1}, x_{2}, \ldots, x_{n}\right\}$ and $Y=\left\{y_{1}, y_{2}, \ldots, y_{m}\right\}$ be the bipartite sets of $K(n, m)$. Let $k \geq 0$ be an integer such that $k 2^{n}+2 \leq m<(k+1) 2^{n}+2$. Then it suffices to show that there exists an edge-coloring of $K(n, m)$ for which the vertices of $K(n, m)$ cannot be covered by $k+1$ vertex-disjoint monochromatic trees.

We color all the edges of $K(n, m)$ red or blue so that $k+1 \leq|Y(r, r, \ldots, r)| \leq$ $|Y(b, b, \ldots, b)| \leq k+2$, and for every $\left(a_{1}, \ldots, a_{n}\right) \neq(r, r, \ldots, r),(b, b, \ldots, b), k \leq$ $\left|Y\left(a_{1}, \ldots, a_{n}\right)\right| \leq k+1$. Note that the number of $n$-tuples $\left(a_{1}, \ldots, a_{n}\right)$ is $2^{n}$, and thus the above edge-coloring exists.

Assume that $V(K(n, m))$ can be covered by $k+1$ vertex-disjoint monochromatic trees $T_{1}, T_{2}, \ldots, T_{k+1}$, where $T_{1}, \ldots, T_{s}$ are trees of order one (i.e., $T_{i}$ consists of one vertex and no edge) and $T_{s+1}, \ldots, T_{k+1}$ are trees of order at least two. Set $F=\left\{T_{1}, T_{2}, \ldots, T_{k+1}\right\}$ and $F^{\prime}=\left\{T_{s+1}, \ldots, T_{k+1}\right\}$.

Let $S=V\left(T_{1}\right) \cup V\left(T_{2}\right) \cup \ldots \cup V\left(T_{s}\right), S_{x}=S \cap X$ and $S_{y}=S \cap Y$, where $|S|=s$. Then $F^{\prime}$ is a set of $k+1-s$ vertex disjoint monochromatic trees, which cover $V(K(n, m))-S$. Let $V_{r}$ and $V_{b}$ denote the subsets of $X-S_{x}$ covered by the red trees and by the blue trees of $F^{\prime}$, respectively. Define the $n$-tuple $\left(a_{1}, a_{2}, \ldots, a_{n}\right)$ by $a_{i}=r$ if $x_{i} \in V_{b} \cup S_{x}$ and $a_{i}=b$ otherwise. It is easy to see that $Y\left(a_{1}, a_{2}, \ldots, a_{n}\right)$ must be contained in $S_{y}$, and so $k \leq\left|Y\left(a_{1}, a_{2}, \ldots, a_{n}\right)\right| \leq\left|S_{y}\right|$.

Hence $\left|F^{\prime}\right|+\left|S_{x}\right|=|F|-\left|S_{y}\right|=k+1-\left|S_{y}\right| \leq k+1-k=1$. This inequality implies that $V_{r}=\emptyset$ or $V_{b}=\emptyset$. Without loss of generality, we may assume $V_{r}=\emptyset$. Then $\left(a_{1}, a_{2}, \ldots, a_{n}\right)=(r, r, \ldots, r), Y(r, r, \ldots, r) \subseteq S_{y}$ and $k+1 \leq|Y(r, r, \ldots, r)|$. This implies $\left|F^{\prime}\right|+\left|S_{x}\right|=|F|-\left|S_{y}\right|=k+1-\left|S_{y}\right| \leq k+1-(k+1)=0$, and hence $|F|=\left|S_{y}\right|$. This is a contradiction.

We are now ready to prove Theorem 1.

Proof of Theorem 1. By Lemmas 2 and 3, we may restrict ourselves to complete multipartite graphs $K\left(n_{1}, n_{2}, \ldots, n_{k}\right)$ with $k \geq 3$. Let $n=n_{1}+n_{2}+\cdots+n_{k-1}$ and $m=n_{k}$.

If $n_{k}=1$, then $\left\lfloor(m-2) / 2^{n}\right\rfloor+2=1 . K\left(n_{1}, n_{2}, \ldots, n_{k}\right)=K(1,1, \ldots, 1)$ is isomorphic to a complete graph, and so it has a monochromatic spanning tree. Thus we may assume $2 \leq n_{k}$. 
We first prove that the set of vertices of 2-edge-colored $K\left(n_{1}, n_{2}, \ldots, n_{k}\right)$ is covered by at most $\left\lfloor(m-2) / 2^{n}\right\rfloor+2$ vertex-disjoint monochromatic trees.

Suppose that $n_{1}+n_{2}+\cdots+n_{k-1}>n_{k} \geq 2$. Then $\left\lfloor(m-2) / 2^{n}\right\rfloor+2=2$. By Lemma $1, K\left(n_{1}, n_{2}, \ldots, n_{k}\right)$ has a spanning complete bipartite graph $K\left(n^{\prime}, m^{\prime}\right)$ such that $n^{\prime} \leq m^{\prime} \leq 2^{n^{\prime}}+1$. Then by Lemma 2 , the vertices of $K\left(n^{\prime}, m^{\prime}\right)$ can be covered by at most two vertex-disjoint monochromatic trees. Hence we may assume that $n=n_{1}+n_{2}+\cdots+$ $n_{k-1} \leq m=n_{k}$.

It is obvious that $K\left(n_{1}, n_{2}, \ldots, n_{k}\right)$ has a spanning complete bipartite graph $K(n, m)$. By Lemma 2, the set of vertices of $K(n, m)$ can be covered by at most $\left\lfloor(m-2) / 2^{n}\right\rfloor+2$ vertex-disjoint monochromatic trees, and so can $K\left(n_{1}, n_{2}, \ldots, n_{k}\right)$.

We next prove that there exists a 2-edge-coloring of $K\left(n_{1}, n_{2}, \ldots, n_{k}\right)$ such that the set of vertices of $K\left(n_{1}, n_{2}, \ldots, n_{k}\right)$ cannot be covered by fewer than $\left\lfloor(m-2) / 2^{n}\right\rfloor+2$ vertex-disjoint monochromatic trees.

Since $n_{k} \geq 2$, we can easily color all the edges of $K\left(n_{1}, n_{2}, \ldots, n_{k}\right)$ red or blue so that the resulting graph has no monochromatic spanning trees. Thus the required statement mentioned above holds if $\left\lfloor(m-2) / 2^{n}\right\rfloor=0$. Hence we may assume that $\left\lfloor(m-2) / 2^{n}\right\rfloor \geq 1$.

It is obvious that $K\left(n_{1}, n_{2}, \ldots, n_{k}\right)$ has a spanning complete bipartite graph $K(n, m)$ with partite sets $V_{1} \cup \ldots \cup V_{k-1}=X$ and $V_{k}=Y$. We color all the edges of $K(n, m)$ in the same way as given in the proof of Lemma 3 , and all the edges of $K\left(n_{1}, n_{2}, \ldots, n_{k}\right)$ not contained in $K(n, m)$ red. Then we apply the same arguments as in the proof of Lemma 3. The condition that there are no edges in $X$ is not necessary for the proof of Lemma 3. Consequently the set of vertices of the 2-edge-colored $K\left(n_{1}, n_{2}, \ldots, n_{k}\right)$ cannot be covered by fewer than $\left\lfloor(m-2) / 2^{n}\right\rfloor+2$ vertex-disjoint monochromatic trees, and hence the theorem is proved.

\section{Proof of Theorem 2}

We simplify notation by denoting by $G$ the complete $k$-partite graph $K\left(n_{1}, n_{2}, \ldots, n_{k}\right)$ with partite sets $V_{1}, V_{2}, \ldots, V_{k}$, where $k \geq 3$. Suppose that all the edges of $G$ are colored red or blue in such a way that for every vertex $v$, at least one red edge and at least one blue edge are incident with $v$.

Let $H$ be the subgraph of $G$ induced by the set of red edges of $G$. Then $H$ is a spanning subgraph of $G$, and we may assume that $H$ is not connected since otherwise $H$ contains a red spanning tree of $G$. Let $D$ be a component of $H$. Then there exist at least two partite sets, say $V_{a}$ and $V_{b}$, which are not covered by $D$ since if $k-1$ partite sets are covered by $D$, then every edge incident with a vertex not contained in $V(D)$ must be blue, which contradicts the assumption that for every vertex, at least one red edge is incident with it. Let

$$
\begin{aligned}
& A=V_{a} \cap V(D) \neq V_{a}, S=V_{a}-A \neq \emptyset, \quad B=V_{b} \cap V(D) \neq V_{b} \\
& T=V_{b}-B \neq \emptyset, X=V(D)-(A \cup B), \quad Y=V(G)-\left(V_{a} \cup V_{b} \cup V(D)\right) . \\
& \text { (see Figure 2) }
\end{aligned}
$$




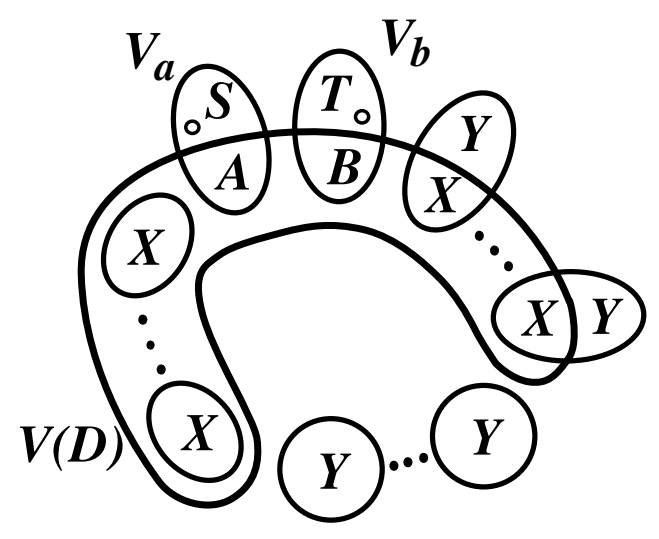

Figure 2: Covering by D

Then since all the edges of $G$ joining a vertex in $V(D)$ to a vertex in $V(G)-V(D)$ are blue, all the edges contained in

$$
E(X, S \cup T) \cup E(A \cup B, Y) \cup E(B, S) \cup E(A, T)
$$

are blue. Thus if $A \cup B \neq \emptyset$ and $X \neq \emptyset$, then $G$ has a blue spanning tree. So we may assume that $A \cup B=\emptyset$ or $X=\emptyset$.

Suppose first $X=\emptyset$. Then since $V(D)=A \cup B \neq \emptyset$ and $D$ has at least one edge, it follows that $A \neq \emptyset$ and $B \neq \emptyset$. Hence $G$ has a blue spanning tree.

We next assume $X \neq \emptyset$. Then $A \cup B=\emptyset$. Let $V_{c}$ be a partite set such that $V_{c} \cap V(D) \neq$ $\emptyset$ and $V_{c} \neq V_{a}, V_{b}$. Let $C=V_{c} \cap V(D) \neq \emptyset$ and $U=V_{c}-C=V_{c} \cap Y$. Then all the edges in $E\left(V_{a} \cup V_{b}, X\right) \cup E(X-C, U) \cup E(C, Y-U)$ are blue. Thus if $X-C \neq \emptyset$, then $G$ has a blue spanning tree, and so we may assume $X-C=\emptyset$, which implies $V(D) \subseteq V\left(V_{c}\right)$. This is a contradiction since $D$ has no edges. Consequently the theorem is proved.

\section{References}

[1] P. Erdös, A. Gyárfás, and L. Pyber, Vertex Coverings by Monochromatic Cycles and Trees, J. Combin. Theory Ser. B 51 (1991), 90-95.

[2] P. E. Haxell, and Y. Kohayakawa, Partitioning by Monochromatic Trees, J. Combin. Theory Ser. B 68 (1996), 218-222.

[3] P. E. Haxell, Partitioning Complete Bipartite Graphs by Monochromatic Cycles, J. Combin. Theory Ser. B 69 (1997), 210-218.

[4] T. Luczak, V. Rödl, and E. Szemerédi, Partitioning 2-Colored Complete Graphs into 2 Monochromatic Cycles, Combinatorics, Probability and Computing 7 (1998), 423-436. 
[5] P. Erdös, and A. Gyárfás, Split and Balanced Colorings of Complete Graphs, Discrete Math 200 (1999), no.1-3, 79-86. 